\title{
Oxycodone regulates incision-induced activation of neurotrophic factors and receptors in an acute post-surgery pain rat model
}

This article was published in the following Dove Press journal: Journal of Pain Research

\section{Baowen Liu \\ Yi Liu \\ Ningbo Li \\ Jin Zhang \\ Xianwei Zhang}

Department of Anesthesiology, Tongji Hospital, Tongji Medical College, Huazhong University of Science and Technology, Wuhan, China
Correspondence: Xianwei Zhang Department of Anesthesiology, Tongji Hospital, Tongji Medical College, Huazhong University of Science and Technology, No 1095 Jie-Fang Road, Wuhan 430030, China

$\mathrm{Tel}+86$ I 3037154560

Fax +86 2783662853

Email ourpain@I63.com
Background: Oxycodone, which is one of the most commonly used opiates in postoperative pain management, has a different affinity for $\mu$-opioid receptors (MOR), $\kappa$-opioid receptors (KOR), and $\delta$-opioid receptors (DOR). Accumulating research has suggested that neurotrophins (NTs) are involved in opioid analgesia. In the current exploratory study, we aimed to investigate the underlying mechanisms of the analgesic effects of oxycodone on post-surgery pain in rats and to determine whether neurotrophic factors and receptors were involved in these effects.

Methods: Mechanical and thermal sensitivity tests were used to evaluate the validity of the postoperative pain rat model and to determine the analgesic effect of oxycodone. Quantitative PCR and Western blot analysis were used to detect the changes in the expression of three types of opioid receptors and NTs and their high-affinity receptors in the spinal cord after surgery and oxycodone administration.

Results: Oxycodone showed an analgesic effect on plantar incision (PI)-induced hyperalgesia, especially thermal hyperalgesia. We detected an obvious increase in MOR expression levels but insignificant changes in KOR and DOR levels in the spinal cord after PI. Moreover, we found that oxycodone was able to reverse the increased expression of nerve growth factor (NGF), brain-derived neurotrophic factor (BDNF), tyrosine kinase receptor $(\operatorname{TrK}) \mathrm{A}$, and $\operatorname{TrkB}$ and the decreased expression of NT-3 and TrkC, after PI. Pretreatment with oxycodone also altered the expression of these mediators.

Conclusion: Based on the results, possible underlying mechanisms for the antinociceptive properties of oxycodone in acute postoperative pain include the activation of MOR downstream signaling and the regulation of NTs and receptor expression through attenuation of glial activation and fortification of antinociceptive mediators in the spinal cord. This study may provide new insights into the molecular mechanisms underlying the analgesic action of oxycodone.

Keywords: oxycodone, acute postoperative pain, opioid receptors, neurotrophins, spinal cord

\section{Introduction}

Acute post-surgery pain after different surgical procedures still remains a significant clinical challenge due to delayed rehabilitation and development of chronic postoperative pain, which is intensely troublesome and difficult to cure. ${ }^{1}$ The need for preventative strategies and effective management of postoperative pain continues to go unmet. Tissue or nerve damage induced by surgical procedures has been shown to result in the activation of nociceptive pathways that lead to neuroinflammation and neuroimmune responses in the spinal cord..$^{2-5}$ Glial cells surrounding the nerve cells produced both pro- and anti-inflammatory cytokines as well as algesic and analgesic mediators such 
as chemokines and neurotrophins (NTs) after activation. ${ }^{6}$ The activation of NTs has been proposed to contribute to hyperexcitability of nociceptive neurons in the spinal cord. ${ }^{7-9}$ NTs are a family that consists of four structurally and functionally related proteins, including nerve growth factor (NGF), brain-derived neurotrophic factor (BDNF), NT-3, and NT-4, which is also known as NT-5 (NT-4 or NT4/5). NTs have an extensive effect on the nervous system; for example, NTs regulate the growth, maintenance, and apoptosis of healthy and injured neurons in the developing nervous system and also regulate survival, proliferation, differentiation, axonal growth, synaptic plasticity, and sensitivity to pain. ${ }^{10,11} \mathrm{NTs}$ are synthesized in dorsal root ganglia (DRG) and transported to the dorsal horn of the spinal cord anterogradely. NTs were activated by binding to two types of receptors, the general neurotrophin receptor (NTR) p75 and neurotrophic tyrosine kinase receptors (Trks). Briefly, all NTs bind to p75NTR with similar affinity, NGF binds to TrkA with high affinity, BDNF and NT-4 bind to TrkB with high affinity, and NT-3 can bind to all NTRs, but with high affinity to TrkC. ${ }^{12}$ It has been described that several gene changes persisted in the primary afferent sensory neurons of the DRGs, following a surgical incision, and then affected nociceptor activation and immune response. Thereinto, gene expression of NGF was increased in incised skin and muscle, which is in agreement with several reports demonstrating the importance of NGF in the development of pain hypersensitivity. ${ }^{13-15}$ Levels of BDNF, another factor that has been shown to increase after surgery, were increased in the spinal cord within primary nerve terminals. ${ }^{9}$ Taken together, it suggested that NTs play an important role in the initiation and maintenance of peripheral sensitization, which leads to subsequent postsurgery pain.

Opioids remain the most effective analgesics for postsurgery pain management despite a host of side effects such as addiction and tolerance. Oxycodone is one of the most commonly used opiates to control mild-to-moderate pain, including postoperative pain. The analgesic effect of oxycodone appears to be 1.5-2.0 times more potent than that of morphine. ${ }^{16}$ However, like most opioids, the most frequently reported side effects of oxycodone are drowsiness, constipation, fatigue, and dizziness. ${ }^{17}$ Oxycodone is a semisynthetic opioid receptor agonist that produces a highly effective analgesic effect on pain by acting on $\mathrm{G}$ protein-coupled opioid receptors. ${ }^{18}$ Although the details of the receptor binding profile remain ambiguous, it has been reported that oxycodone has a relatively high affinity for $\mu$-opioid receptors (MORs), relatively little affinity for $\kappa$-opioid receptors (KORs), and little to no affinity for $\delta$-opioid receptors (DORs). ${ }^{19-21}$ Since various cells in the central and peripheral nervous system, immune system, and neuroendocrine system express opioid receptors, their function can be modulated by endogenous and exogenous opioids. ${ }^{22,23}$ Miranda et al, using in vivo electrophysiology, found that oxycodone reduced the response of spinal dorsal horn neurons to noxious knee joint rotation in monosodium iodoacetate-sensitized rats, suggesting a regulatory effect of oxycodone in the spinal cord. ${ }^{24} \mathrm{~A}$ previous study by Hassan et al that involved microarray profiling and gene mapping analysis clarified that oxycodone indeed modulated gene expression and regulated many biological processes such as immune response and the transmembrane receptor tyrosine kinase signaling pathway in rats repeatedly treated with oxycodone..$^{25}$ A recent study used unbiased RNA sequencing to examine gene expression in a mouse model of 14-day oxycodone self-administration and found that oxycodone administration altered the expression of numerous inflammatory and immune-related genes in the striatum. In particular, gene expression of NGF receptor was significantly downregulated in the ventral striatum after chronic oxycodone administration. ${ }^{26}$ In addition, a recent study suggested that opiate agonists can attenuate the upregulation of BDNF in the DRG and the spinal cord in a rat surgical pain model. ${ }^{27}$ However, the effect of oxycodone on NTs and receptors in the spinal cord needs further investigation.

Although the abirritation of oxycodone on post-surgery pain is well known, the mechanisms underlying the effects of oxycodone in the incision-sensitized spinal cord are poorly understood. Accumulating evidence indicates that NTs have essential modulatory roles in opioid analgesia. ${ }^{28}$ Given the remarkable function of NTs in opioid analgesia, it is crucial to determine if the expression of these mediators in the spinal cord is regulated by oxycodone. The current exploratory experiment aimed to identify which opioid receptor subtypes contributed to the effects of oxycodone on post-surgery pain in rats and whether oxycodone has an effect on NTs and their high-affinity receptors.

\section{Materials and methods}

\section{Animal care}

All experiments were performed on Sprague Dawley rats (weight: 250-300 g), which were raised in the animal center of Tongji Hospital. All animals were housed in group cages with rodent chow and water available ad libitum. Rats were habituated to their environment (temperature: $22^{\circ} \mathrm{C}-24^{\circ} \mathrm{C}$; humidity: $50 \%-60 \%$; light: 12 -hour light/ dark cycle) at least 3 days before the experiments. All 
experiments were performed using protocols approved by the Institutional Animal Care and Use Committee of Tongji Hospital, Huazhong University of Science and Technology in accordance with the National Institutes of Health Guide for the Care and Use of Laboratory Animals (NIH Publications No 80-23), revised in 1996. The study is also approved by the ethical committee of Tongji Hospital, Tongji Medical College, Huazhong University of Science and Technology.

\section{Animal behavioral test}

Three days before the test, rats were singly placed in Plexiglas chambers, which were elevated by a wire mesh or glass floor, for 30 minutes daily to acclimatize to the testing environment. Mechanical sensitivity was assessed by an electronic Von Frey filament (Model 38450; Ugo Basile, Gemonio, Italy). Measurements ranged from 0 to $200 \mathrm{~g}$. The paw withdrawal threshold (PWT) was measured by applying the filament to the plantar surface of the right hind paw vertically, adjacent to the wound. Thermal sensitivity was assessed using the Hargreaves Apparatus (Model 37370; Ugo Basile). The paw withdrawal latency (PWL) was determined by applying an infrared radiant heat beam focused on the plantar area of the right hind paw. The intensity of heat was adjusted to produce baseline latencies of 15-20 seconds in normal rats. The infrared value used in this experiment was 95 units. The cutoff time was set at 30 seconds to avoid tissue damage. All stimuli were conducted when animals were calm but not grooming or sleeping, and data were calculated from the average of the three tests.

\section{Acute postoperative pain model}

The plantar incision (PI) surgery was performed as previously described. ${ }^{29}$ Briefly, after anesthetized with $2 \%$ isoflurane through a nose cone, the surface of the right hind paw was prepared under sterile conditions. A $1 \mathrm{~cm}$ incision was made longitudinally through the skin and fascia of the surface of the right hind paw, beginning $0.5 \mathrm{~cm}$ from the proximal edge of the heel toward the toes. Keeping the muscles' origin and insertion intact, the flexor muscle was elevated and incised. Gentle pressure was applied for hemostasis. A mattress suture of 5-0 nylon was used to suture the incision. An antibiotic was applied to the wound. The rats in the control group received anesthesia but did not receive an incision.

\section{Experimental design}

The rats were randomly assigned to four groups, each with eight animals: 1) control group, ie, after anesthesia, $2 \mathrm{~mL} / \mathrm{kg}$ of saline was administered intraperitoneally; 2) PI group, ie, after anesthesia, a PI was performed on the right hind paw and then $2 \mathrm{~mL} / \mathrm{kg}$ of saline was administered intraperitoneally; 3) postoperative oxycodone group (PI+OXY), ie, single oxycodone (2 mg/kg diluted in saline; 30044090.40, Mundipharma, Beijing, China) was administered intraperitoneally 15 minutes after incision; and 4) preoperative oxycodone group $(\mathrm{OXY}+\mathrm{PI})$, ie, $2 \mathrm{mg} / \mathrm{kg}$ of oxycodone was intraperitoneally injected preoperatively. Fifteen minutes after drug administration, the animals underwent incision surgery. Baseline assessments of withdrawal thresholds were performed prior to surgery for 3 days. Afterward, the mechanical and thermal withdrawal thresholds of all incision groups were measured at $1,2,4,6,8,12$, and 24 hours after incision, while the control group was examined after recovery from anesthesia. After the last behavioral test, the rats were sacrificed under deep anesthetic conditions, and tissue samples were collected for further analysis. Following decapitation, the spinal columns were opened to expose the lumbar spinal cord. The lumbar enlargement of the spinal cord was cut, collected rapidly, and stored at $-80^{\circ} \mathrm{C}$ for subsequent analysis after freezing in liquid nitrogen.

\section{Real-time quantitative PCR (RT-qPCR)}

Total RNA was isolated from the lumbar enlargement of the spinal cord with RNAiso Plus kit (Takara, Dalian, China) using the manufacturer's recommended protocols. Spectrophotometric analysis of the total RNA was conducted using a Nano Drop Lite (Thermo Fisher Scientific, Waltham, MA, USA). The samples with an A260/A280 ratio greater than 1.7 were reverse transcribed to synthesize cDNA using the Prime Script RT reagent Kit (Takara) according to the manufacturer's directions. RT-qPCR was performed using a StepOne machine (StepOne; Thermo Fisher Scientific) with SYBR Premix Ex Taq II (Takara). Each gene was run to obtain dissociation curves to test the amplicon specificity. The primer sequences were as follows (forward and reverse): MOR: 5'-GCCCTCTATTCTATCGTGT-3' and 5'-TATGGCTAAGGCATCTGC-3'; NGF: 5'-CATCGCTCTCCTTCACAGAGTT-3' and 5'-TGCCTGTACGCCGATCAAAA-3'; BDNF: 5'-TTACCTGGATGCCGCAAACA-3' and 5'-TGGCCTTTTGATACCGGGAC-3'; NT-3: 5'-CCTCAGCCATTGACATTCGG-3' and 5'-TCAGTGCTCGGACGTAGGTTT-3'; TrkA: 5'-GCATCCTGTCGTGAGACCTG-3' and 5'-AGATCCCGCTGGTTTTCCAC-3'; TrkB: 5'-ACCAAACCAATCGGGAGCAT-3' and 5'-CTGGGCCTTTCATGCCAAAC-3'; TrkC: 5'-ACATCGCCAGTCAGATAGCC-3' and 5'-TGATGCTGCTAGCCTCTGTT-3'; and GAPDH: 5'-ACAGCAACAGGGTGGTGGAC-3' and 5'-TTT 
GAGGGTGCAGCGAACTT-3'. Data were quantified by the comparative CT method with the mathematical formula as described previously: $\Delta \mathrm{Ct}=\mathrm{Ct}$ (gene of interest) $-\mathrm{Ct}$ (housekeeping gene); $\Delta \Delta \mathrm{Ct}=\Delta \mathrm{Ct}-$ average $\Delta \mathrm{Ct}$ (control group); and fold change $=2^{\wedge(-\Delta \triangle \mathrm{CT})}$.

\section{Western blot analysis}

The lumbar enlargement of the spinal cord was homogenized in a RIPA buffer and protease inhibitor cocktail. The homogenates were centrifuged at $12,000 \times g$ for 15 minutes at $4^{\circ} \mathrm{C}$. Protein concentrations were determined by Pierce ${ }^{\mathrm{TM}}$ BCA Protein Assay Kit (Thermo Fisher Scientific). The supernatants of the homogenates were boiled at $100^{\circ} \mathrm{C}$ in loading sample buffer for 5 minutes. The samples contained $35 \mu \mathrm{g}$ proteins, were electrophoresed on 10\%-12\% SDS/PAGE gel, and then transferred to polyvinylidene fluoride membranes (EMD Millipore, Billerica, MA, USA). Membranes were first blocked with $5 \%(\mathrm{w} / \mathrm{v})$ defatted milk in $0.1 \%$ Tween 20 (TBST; $2 \mathrm{mmol} / \mathrm{L}$ Tris- $\mathrm{HCl}, 50 \mathrm{mmol} / \mathrm{L} \mathrm{NaCl}, \mathrm{pH}$ 7.4) for 2 hours at room temperature and followed by incubation overnight at $4{ }^{\circ} \mathrm{C}$ with specific primary antibody for OPRM1 (1:1,000, A7264; ABclonal, Wuhan, China), NGF (1:1,000, ab52918; Abcam, Cambridge, UK), BDNF (1:500, DF6387; Affinity, Whhan, China), NT-3 (1:1,000, DF6105; Affinity), TrkA (1:200, BA0404; Boster, Wuhan, China), TrkB (1:200; Affinity), and TrkC (1:500, A14033; ABclonal, Wuhan, China). After being thoroughly rinsed with TBST, membranes were incubated with HRP-conjugated goat anti-rabbit (EMD Millipore) or goat antimouse secondary antibody (EMD Millipore; diluted in 1:5,000) for 2 hours at room temperature. After being thoroughly washed with TBST, the specific antibody binding was visualized using the ECL system (Thermo Fisher Scientific). The protein bands were quantified based on gray value using an image analysis software (Image Lab) and normalized to $\beta$-actin.

\section{Drugs}

The Oxycodone Hydrochloride Injection was obtained from Beijing Mundipharma Pharmaceutical Co., Ltd. (Beijing, China).

\section{Statistics and data analysis}

Results were expressed as mean \pm standard error of the mean (SEM) and error bars represented SEM. Behavioral tests were performed using a two-way ANOVA with repeated measures, followed by post hoc Bonferroni tests. The expressions values of genes and proteins were analyzed using a one-way ANOVA for multiple comparisons, followed by post hoc Bonferroni tests. All data were analyzed with GraphPad Prism 5.01 software (GraphPad Software, Inc., La Jolla, CA, USA). A value of $P<0.05$ was considered statistically significant.

\section{Results}

\section{Behavioral experiments}

As shown in Figure 1, there were no significant differences in baseline mechanical and thermal withdrawal thresholds among groups $(P>0.05)$. The mechanical withdrawal threshold and thermal withdrawal latency were significantly decreased after PI in the PI group from 1 hour $(7.5 \pm 0.47 \mathrm{~g}$; $7.7+0.61$ seconds $)$ to 24 hours $(10.7 \pm 1.02 \mathrm{~g} ; 13 \pm 0.94 \mathrm{sec}-$ onds), indicating that incision surgery induced hyperalgesia of the right hind paw. The results of the behavioral tests showed that the maximum analgesic effect of single oxycodone administration, postoperatively or preoperatively, was reached within the first 2 hours (PI+OXY: 1 hour: $54.8 \pm 9.39 \mathrm{~g}$

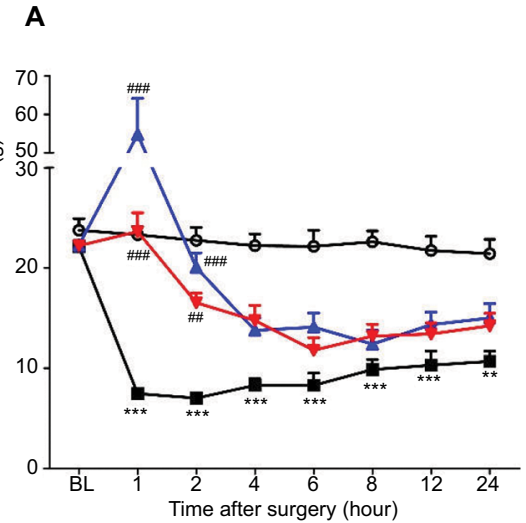

B

Figure I Mechanical and thermal hyperalgesia induced by PI and the analgesic effect of oxycodone.

Notes: (A) The mechanical withdrawal thresholds. (B) The thermal withdrawal thresholds. ${ }^{* * P} P<0.01$, ${ }^{* * *} P<0.00$ I vs control group; ${ }^{\#} P<0.05$, ${ }^{\# P} P<0.0$ I, ${ }^{\#} P<0.00$ I vs $P I$ group (two-way ANOVA followed by Bonferroni's multiple comparison post hoc test, $\mathrm{n}=8$ rats per group). Each value represents mean $\pm \mathrm{SEM}$. $\mathrm{PI}+\mathrm{OXY}$, postoperative oxycodone group; OXY+PI, preoperative oxycodone group.

Abbreviations: BL, baseline; PI, plantar incision; SEM, standard error of the mean. 
and $30.1 \pm 0$ seconds; 2 hours: $20.1 \pm 1.43 \mathrm{~g}$ and $21.4 \pm 2.14 \mathrm{sec}-$ onds; OXY+PI: 1 hour: $23.6 \pm 1.87 \mathrm{~g}$ and $20.5 \pm 0.84$ seconds; and 2 hours: $16.5 \pm 0.94 \mathrm{~g}$ and $19.4 \pm 0.87$ seconds) (Figure 1).

\section{The expression of MOR was upregulated in the spinal cord after PI}

We assayed mRNA and protein expression of three opioid receptors (MOR, DOR, and KOR) in the spinal cord 24 hours post-surgery using quantitative PCR and Western blotting. We did not detect significant changes in DOR and KOR gene and protein expressions in $\mathrm{PI}, \mathrm{PI}+\mathrm{OXY}$, and $\mathrm{OXY}+\mathrm{PI}$ groups after surgery, compared to the control group (data not shown). We did, however, observe an increase in MOR levels after PI in PI, PI+OXY, and OXY+PI groups when compared to the control group (Figure 2A: $P=0.0014, F=10.04$; Figure 2B: $P=0.0025, F=8.653$ ). Compared to the PI group, there was an insignificant increase in MOR expression after oxycodone administration $(P>0.05)$.

\section{Altered expression of NTs in the spinal cord 24 hours after PI and oxycodone application}

We measured mRNA and protein expressions of NTs (NGF, BDNF, NT-3, and NT-4). We found distinct expression profiles of NTs after PI and oxycodone administration. The mRNA and protein expressions of NGF and BDNF showed a remarkable upregulation, and NT-3 showed a remarkable downregulation after surgery, whereas oxycodone showed an inhibition effect on NGF (Figure 3A: $P=0.000, F=20.85$;

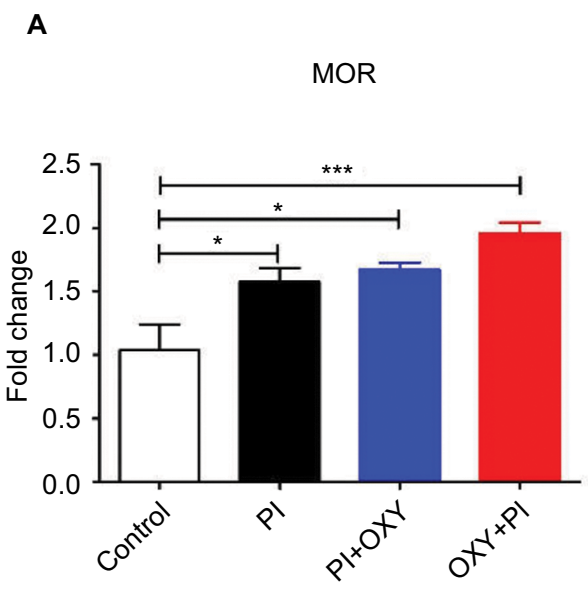

Figure 3B: $P=0.0002, F=14.87$ ) and BDNF (Figure 3C: $P=0.0091, F=6.403$; Figure 3D: $P=0.000, F=64.05)$ and an elevation effect on NT-3 (Figure 3E: $P=0.0006, F=14.33$; Figure 3F: $P=0.000, F=49.15$ ) both in gene and protein levels. We did not detect significant changes in gene and protein expression of NT-4 (data not shown).

\section{Altered expression of neurotrophic receptors in the spinal cord 24 hours after PI and oxycodone application}

As shown in Figure 4, we measured mRNA and protein expression of high-affinity receptors of NTs, including TrkA, TrkB, and TrkC. Statistical comparison between the PI group and the control group showed that the mRNA and protein expression of TrkA and TrkB in the PI group were significantly higher than that of the control group, while the expression of TrkC was decreased. When statistically compared with the PI group, TrkA (Figure 4A: $P=0.0011$, $F=11.23$; Figure 4B: $P=0.0007, F=17.72$ ) and TrkB (Figure 4C: $P=0.0003, F=17.61$; Figure 4D: $P=0.0004, F=13.12$ ) expressions in the $\mathrm{PI}+\mathrm{OXY}$ and $\mathrm{OXY}+\mathrm{PI}$ groups were significantly lower. Conversely, the expression of TrkC (Figure 4E: $P=0.0027, F=8.972$; Figure 4F: $P=0.000, F=32.92$ ) displayed a noticeable increase in the PI+OXY and OXY+PI groups.

\section{Oxycodone suppresses the activation of glial cells in the spinal cord}

The mRNA levels of GFAP and Iba1 after 24 hours of PI were significantly upregulated in the PI group and were

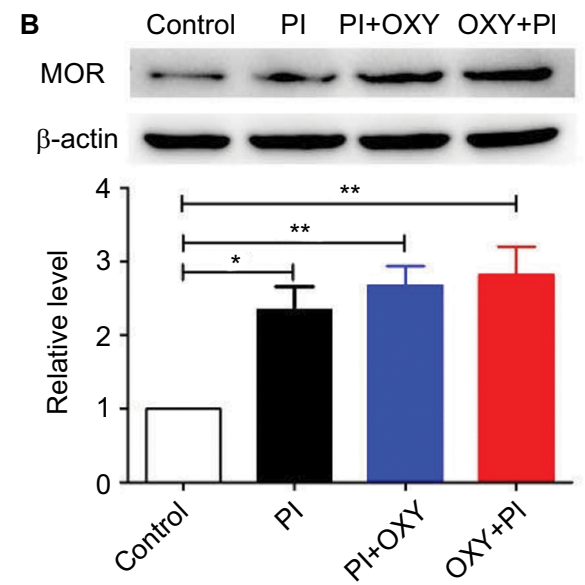

Figure $2 \mathrm{Pl}$ induced the activation of MOR in the spinal cord.

Notes: (A) The mRNA levels of MOR were significantly increased in PI, PI+OXY, and OXY+PI groups. (B) The upper panel represents results of the Western blot analysis. The lower panel shows the semi-quantitative analysis of MOR after normalization to the corresponding $\beta$-actin. The expression of MOR was significantly increased in PI, $\mathrm{PI}+\mathrm{OXY}$, and $\mathrm{OXY}+\mathrm{PI}$ groups $\left(* \mathrm{P}<0.05\right.$, ${ }^{*} * \mathrm{P}<0.0 \mathrm{I}$, ${ }^{* * * P}<0.00 \mathrm{I}$ vs control group, one-way ANOVA followed by Bonferroni's multiple comparison post hoc test, $\mathrm{n}=3-4$ rats per group). Each value represents mean $\pm \mathrm{SEM}$. $\mathrm{PI}+\mathrm{OXY}$, postoperative oxycodone group; OXY+PI, preoperative oxycodone group.

Abbreviations: MOR, $\mu$-opioid receptor; PI, plantar incision; SEM, standard error of the mean. 
A

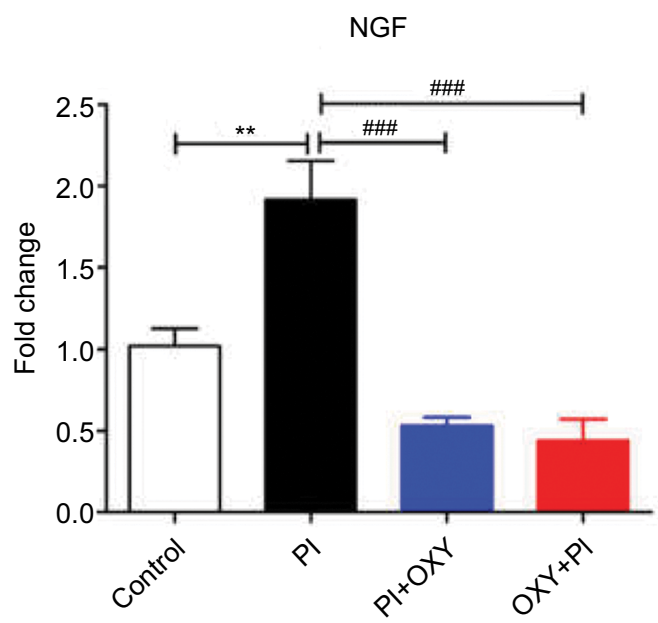

C

BDNF

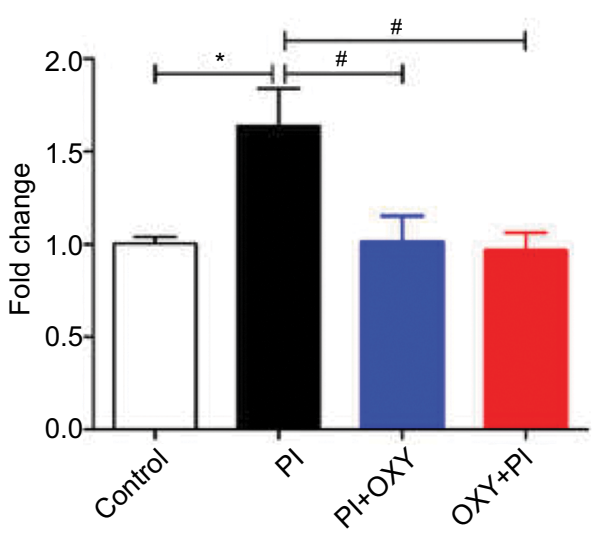

E

NT-3

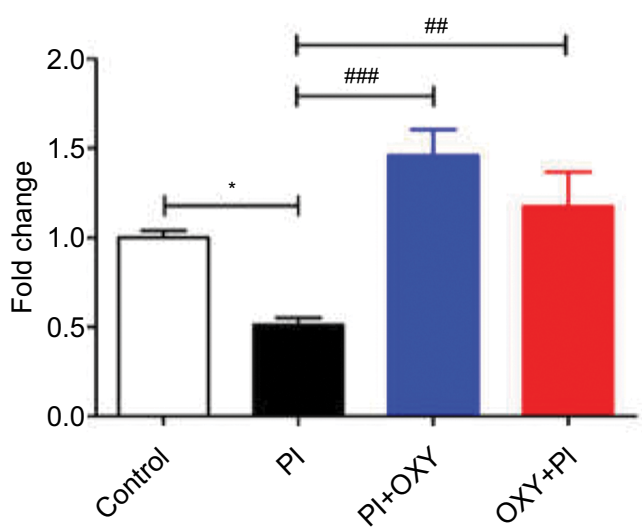

B

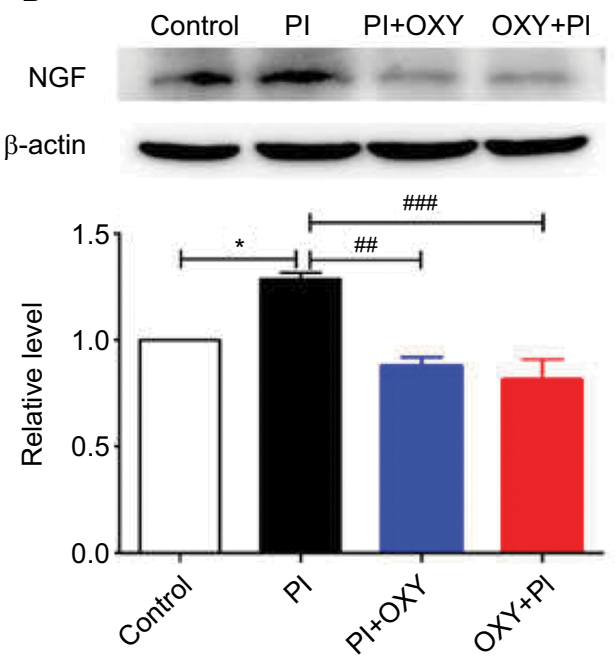

D
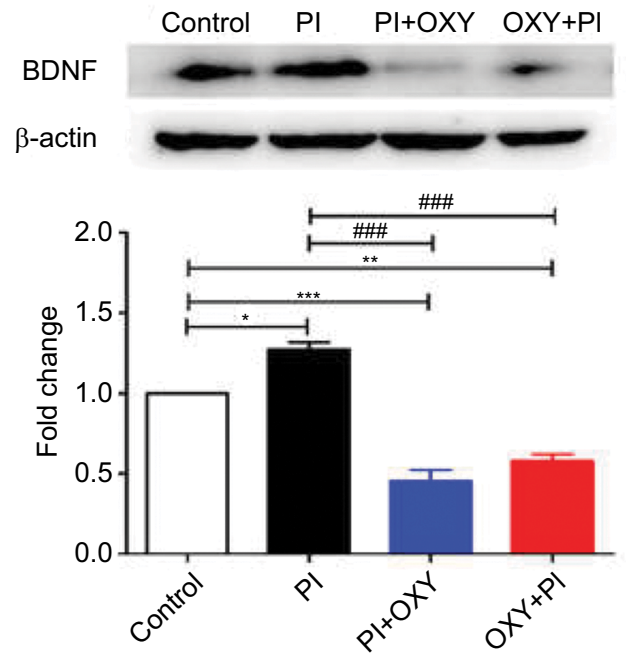

F

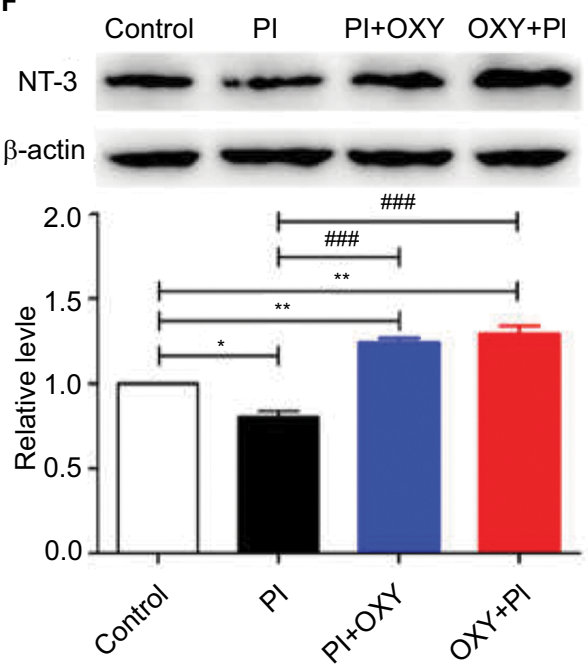

Figure 3 The altered expression of NTs in the spinal cord 24 hours after PI and the effects of oxycodone on NTs.

Notes: (A, C, and E) The mRNA level of NGF, BDNF, and NT-3. (B, D, and F) The upper panels represent results of the Western blots. The lower panels show the semiquantitative analysis of NGF, BDNF, and NT-3 after normalization to the corresponding $\beta$-actin $\left(* P<0.05, * * P<0.01\right.$, ${ }^{* * *} P<0.00$ I vs control group; ${ }^{*} P<0.05$, $P<0.0$ I, ${ }^{*} P<0.00$ I vs $\mathrm{PI}$, one-way ANOVA followed by Bonferroni's multiple comparison post hoc test, $\mathrm{n}=3-4$ rats per group). Each value represents mean $\pm \mathrm{SEM}$. $\mathrm{PI}+\mathrm{OXY}$, postoperative oxycodone group; OXY+PI, preoperative oxycodone group.

Abbreviations: BDNF, brain-derived neurotrophic factor; NGF, nerve growth factor; NT, neurotrophin; PI, plantar incision; SEM, standard error of the mean. 
A

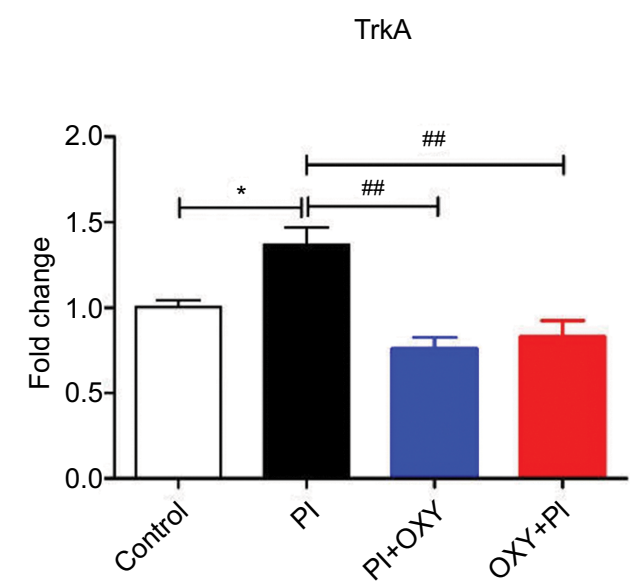

C

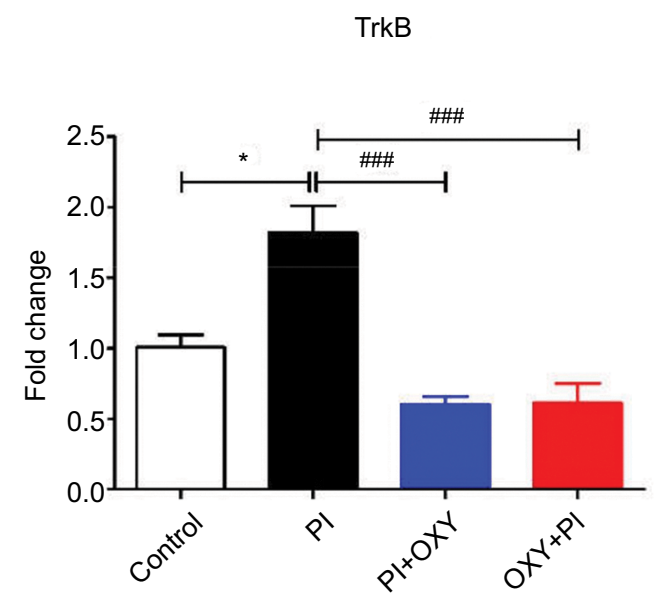

E

TrkC

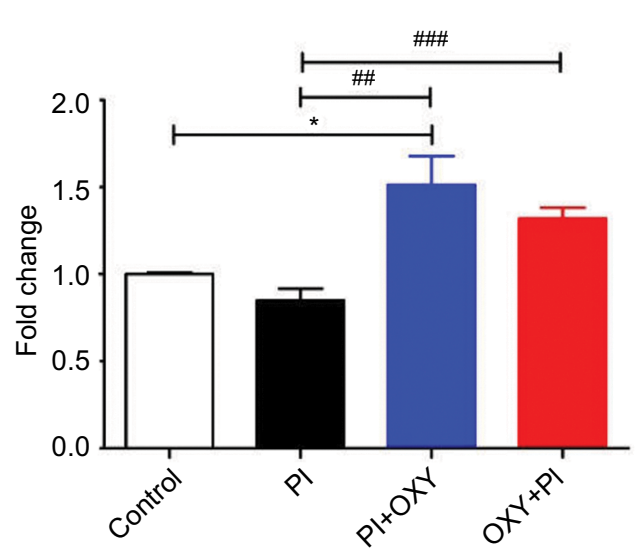

B

Control

$\mathrm{PI}$

$\mathrm{PI}+\mathrm{OXY} O \mathrm{OXY}+\mathrm{PI}$

TrkA

$\beta$-actin

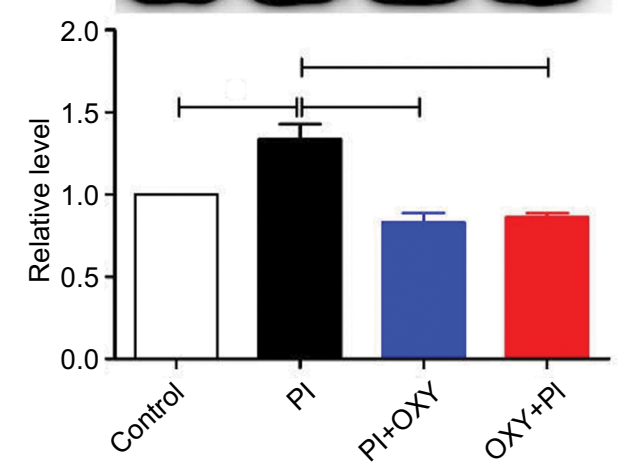

D

TrkB Control PI PI+OXY OXY+PI
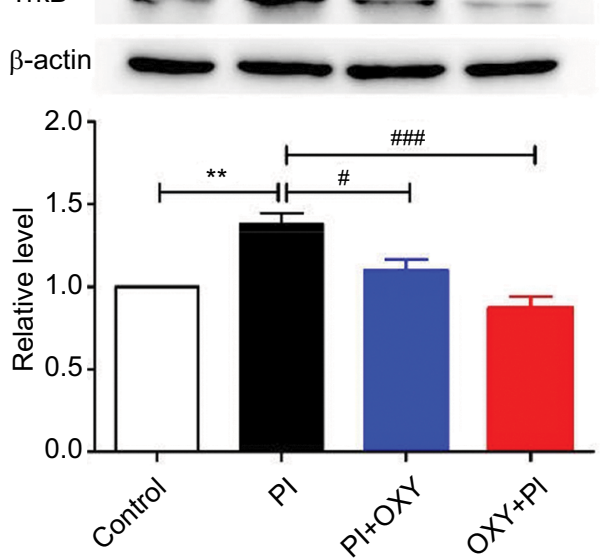

$\mathbf{F}$

F Control PI $\quad \mathrm{PI}+\mathrm{OXY} \quad \mathrm{OXY}+\mathrm{PI}$

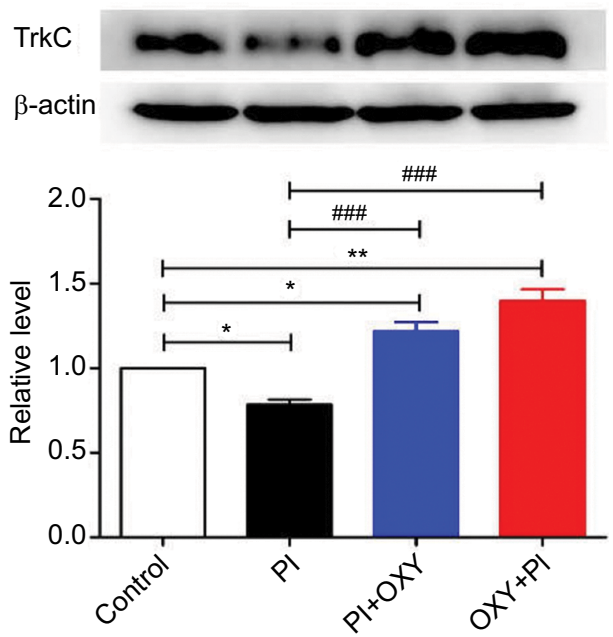

Figure 4 The altered expression of neurotrophic receptors in the spinal cord 24 hours after PI and the effects of oxycodone on neurotrophic receptors.

Notes: (A, C, and E) RT-qPCR was performed to examine the mRNA level of TrkA, TrkB, and TrkC. (B, D, and F) The upper panels represent results of the Western blots. The lower panels show the semi-quantitative analysis of TrkA, TrkB, and TrkC after normalization to the corresponding $\beta$-actin. $(* P<0.05$, $* * P<0.01$ vs control group; ${ }^{\#} P<0.05,{ }^{\#} P<0.01,{ }^{\#} P<0.001$ vs $\mathrm{PI}$, one-way ANOVA followed by Bonferroni's multiple comparison post hoc test, $n=3-4$ rats per group). Each value represents mean $\pm S E M$. $\mathrm{PI}+\mathrm{OXY}$, postoperative oxycodone group; $\mathrm{OXY}+\mathrm{PI}$, preoperative oxycodone group.

Abbreviations: PI, plantar incision; RT-qPCR, real-time quantitative PCR; SEM, standard error of the mean; Trks, tyrosine kinase receptors. 
downregulated in the PI+ OXY and OXY+PI after oxycodone application (Figure 5A: $P=0.000, F=19.31$; Figure 5B: $P=0.0017, F=12.01)$.

\section{Discussion}

Although a considerable number of drugs are used for clinical analgesia, acute post-surgery pain is still highly prevalent. Surgical procedures in patients usually include skin incisions and muscle separation and retraction to remove inflamed organs or tumors. If an experimental model can mimic the etiology and pathophysiology of a disease, then the underlying mechanisms may be better understood and the treatments may be improved. For PI in rats, the paw skin and fascia were incised, and underlying flexor muscle was elevated and subsequently incised. Thus, the injury after PI mimicked the somatic injury of many patients who undergo surgery. In our results, mechanical and thermal hyperalgesia were present on the affected limb within 1 hour and persisted for 24 hours post-surgery in the PI group. Both mechanical and thermal hyperalgesia are present in surgery patients. ${ }^{30,31}$ According to previous studies and our preliminary experiment, incision-induced mechanical and heat hyperalgesia were greatest the day of the incision. Thus, we chose 24 hours after incision as the experiment end point. ${ }^{13}$ Primary afferent activation and peripheral sensitization were profound early after surgery and most changes in protein expression occurred when pain behavior was most pronounced..$^{32,33}$ Therefore, we examined the neurobiological changes in the spinal cord 24 hours post-surgery. We intraperitoneally administered a moderate dosage $(2 \mathrm{mg} / \mathrm{kg})$ of oxycodone in PI+ OXY and OXY+PI groups 15 minutes post- and pre-surgery. A moderate dose
A

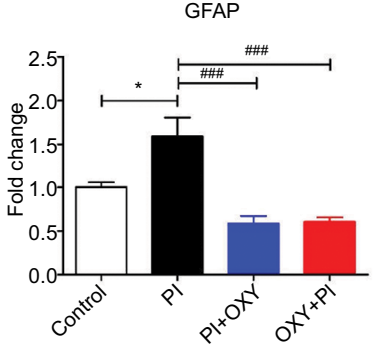

B

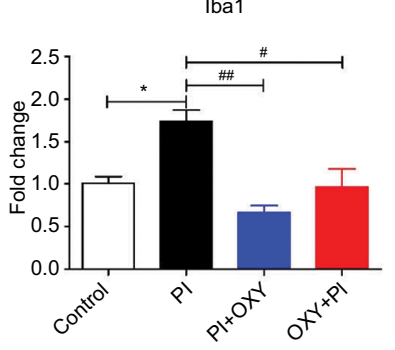

Figure 5 The inhibiting effect of oxycodone on glial activation. Notes: (A) The mRNA level of GFAP was significantly increased in $\mathrm{Pl}$ and decreased in $\mathrm{PI}+\mathrm{OXY}$ and $\mathrm{OXY}+\mathrm{PI}$ groups. (B) The mRNA level of lbal was significantly increased in the $\mathrm{PI}$ group and decreased in the $\mathrm{PI}+\mathrm{OXY}$ and $\mathrm{OXY}+\mathrm{PI}$ groups ( ${ }^{*} P<0.05$ vs control; ${ }^{\# P} P 0.05,{ }^{\# P} P<0.01,{ }^{\# *} P<0.00$ I vs $P$, one-way ANOVA followed by Bonferroni's multiple comparison post hoc test, $n=3-4$ rats per group). Each value represents mean $\pm \mathrm{SEM}$. $\mathrm{PI}+\mathrm{OXY}$, postoperative oxycodone group; $\mathrm{OXY}+\mathrm{PI}$, preoperative oxycodone group.

Abbreviations: GFAP, glial fibrillary acidic protein; Ibal, ionized calcium-binding adaptor molecule I; PI, plantar incision; SEM, standard error of the mean. of oxycodone was chosen to avoid opioid-induced hyperalgesia and central nervous system depression. ${ }^{34,35}$ Oxycodone induced a reversal of mechanical hyperalgesia in rats that had been subjected to surgery; the maximal reversal lasted for 2 hours post-administration. However, oxycodone reduced and gradually eliminated thermal hyperalgesia up to the end point. Pretreatment with oxycodone also had an impact on mechanical and thermal hyperalgesia, with a lower peak effect than with posttreatment. Similar to humans, in rats, mechanical sensitivity requires a considerably longer time to resolve than heat hypersensitivity. ${ }^{36,37}$ The differences in recovery time between heat sensitivity and mechanical sensitivity indicated distinct neuronal pathways.

We assayed the mRNA and protein levels of three opioid receptors in the spinal cord following incision and administration of oxycodone. Compared to the control group, our results indicated enhanced MOR levels after PI. However, oxycodone acts as an opioid receptor agonist and exerts its effect via activating opioid receptors without affecting the expression of opioid receptors. Therefore, compared to the PI group, there were insignificant changes in the expression of opioid receptors after the administration of oxycodone in $\mathrm{PI}+\mathrm{OXY}$ and $\mathrm{OXY}+\mathrm{PI}$ groups. Previous studies have shown that inflammation results in an enhanced MOR G-protein coupling and MOR agonist efficacy by increasing MOR binding and immunoreactivity within DRG. ${ }^{38-40}$ Our results are consistent with the theory that tissue injury activates pain inhibitory systems following the increased expression of MOR to limit the intensity and duration of pain hypersensitivity associated with the incision. ${ }^{41}$ The upregulated expression of MOR after injury resulted in enhanced opioid susceptibility. Most importantly, we did not detect changes in the expression of KOR and DOR after PI, suggesting that oxycodone's analgesic effect acts on MOR. It has been reported that the analgesic effect of oxycodone in some pain models may be mediated through activation of the MOR. ${ }^{42,43}$ Furthermore, the antinociceptive effect was antagonized by naloxone but not antagonized by selective $\kappa$-antagonist norbinaltorphimine. ${ }^{43}$ Consistent with previous studies, ${ }^{42,43}$ our results indicate that the effect of oxycodone in rat incisioninduced pain can be attributed to the activation of MOR.

Next, we examined the mRNA and protein levels of all NTs and their high-affinity receptors in the spinal cord following incision and administration of oxycodone. In our results, compared to the control group, the expression of NGF, BDNF, and their high-affinity receptors TrkA and TrkB were increased in the spinal cord post-surgery. NTs can transport from the lumbar DRG into spinal cord 
anterogradely, suggesting that they may play a role in the modulation of nociceptive signaling in the spinal cord. ${ }^{44,45}$ It is well known that NGF and its interaction with TrkA are critical mediators of pain initiation and maintenance. ${ }^{7}$ NGF levels were reported to be elevated in inflammation, peripheral nerve injury, and neuropathic pain, and NGFsequestering antibodies are effective in many pain models. In addition, an increasing number of studies are providing evidence for the functional role of NGF in the activation and sensitization of nociceptors in rodents and humans via intradermal injection. ${ }^{46-48}$ NGF also led to transcriptional changes through the binding of TrkA, causing the elevated expression of pro-nociceptive neurotransmitters such as substance P (SP), calcitonin gene-related peptide (CGRP), and BDNF, which were released after nociceptors were activated and acted as a central modulator. ${ }^{46,49} \mathrm{It}$ has been well characterized that the critical role of spinal BDNF involves inflammatory and neuropathic pain. ${ }^{9,50}$ The expression of BDNF peaked at 24-48 hours in the spinal dorsal horn in the rat neuropathic pain model. Furthermore, neutralizing the increased expression of spinal BDNF could alleviate pain. ${ }^{51}$ In this study, on the one hand, the elevated expression of BDNF may be caused by the activation of nociception after PI or it may be due to increased NGF. NT-4, a ligand for TrkB such as BDNF, is synthesized in DRGs and expressed predominantly by motor neurons in the ventral horn of the spinal cord. NT-4 plays a role in the maintenance and survival of motor neurons, which is very different from the role of BDNF. ${ }^{52-55}$ Moreover, several studies have provided evidence to support that NT-4 may play a less significant role in nociceptive transmission and neuropathic pain than other NTs, but this finding requires further investigation. ${ }^{53,55,56}$ In parallel with these reports, we did not detect changes in NT-4 expression in our rat model of post-surgery pain. After applying oxycodone in $\mathrm{PI}+\mathrm{OXY}$ and $\mathrm{OXY}+\mathrm{PI}$ groups post- and pre-surgery, the increased expression of NGF, BDNF, TrkA, and TrkB was reversed, demonstrating that oxycodone could mediate the expression of NTs and receptors in acute surgery pain in rats. We found that pretreatment with oxycodone could also alter the expression of these mediators, suggesting that a prophylactic drug that reduces the magnitude of postoperative pain could be useful. Joseph and Levine ${ }^{57}$ demonstrated that MOR localized on a functionally important population of TrkA-positive nociceptors, suggesting that MOR played a regulated role in NGF-induced hyperalgesia. This finding indicated that oxycodone plays a role in regulating NT and receptor expression. Accordingly, the second possible mechanism we conjecture is that the antinociceptive effect of oxycodone in acute post-surgery pain may involve the regulation of NTs and receptors.

Nevertheless, the underlying mechanism of NT regulation by oxycodone warrants further investigation. According to classical opioid-induced signaling, oxycodone acted as an opioid agonist and activated MOR in nociceptors, followed by the inhibition of cyclic adenosine monophosphate (cAMP) and the decreased production of downstream proteins including NTs and receptors. However, many opioids also acted through non-opioid receptors and novel modulatory mechanisms. It is well known that the injury of both peripheral and central nerves gives rise to the activation of astrocytes and microglia. ${ }^{58}$ Microglia are the main immune cell type in the central nervous system, which change in morphology and synthesize several mediators during neuronal injury. ${ }^{59,60}$ Both activated astrocytes and microglia can secrete pro- and antiinflammatory cytokines, chemokines, neurotrophic factors, and many other molecular modulators. ${ }^{61}$ Oxycodone could suppress the glial activation and pro-inflammatory cytokines in mice models of neuropathic pain. ${ }^{62}$ Most importantly, the inhibiting effect of oxycodone on glial activation takes place in the early stages of drug administration. ${ }^{62} \mathrm{~A}$ recent study showed that medium and high concentrations of oxycodone could regulate the pro-inflammatory and anti-inflammatory molecules released by activated microglia in vitro. ${ }^{63} \mathrm{We}$ also examined the mRNA levels of GFAP and Iba1, which are astrocytic and microglial markers, respectively. In parallel with these studies, our results showed that oxycodone had an inhibitory effect on glial activation, suggesting that the effect of oxycodone on NTs may be mediatory by suppressing the activation of glial cells. NTs have been reported to lead to both pro-nociceptive and antinociceptive effects, suggesting a complex mechanism involving NTs in initial pain and pain maintenance. ${ }^{64-66} \mathrm{NT}-3$, another NT, acted in a manner that antagonized nociceptive phenotypes in sensory neurons. Applying NT-3 to neuropathic pain in rats could prevent and attenuate thermal hyperalgesia in injured hind paws with a negative effect on mechanical hyperalgesia. ${ }^{45}$ Our data showed that the expression of NT-3 and its highaffinity receptor TrkC were decreased in the spinal cord in the PI group, post-surgery, and after applying oxycodone, the expression of NT-3 and TrkC were increased. A study revealed that NT-3 functionally reduced the expression of TrkA, BDNF, transient receptor potential vanilloid receptor-1 (TRPV1), SP, and CGRP in intact neurons, demonstrating 
a beneficial role of NT-3 in inhibiting the pro-nociceptive effect of NGF. ${ }^{45}$ Interestingly, another study observed that the inhibition of SP release by NT-3 in the spinal cord of rats could be reversed with naloxone, suggesting the presence of cross-talk between opioidergic signaling and NT-3 in the spinal cord. ${ }^{67}$ Thus, we propose that oxycodone may upregulate the expression of NT-3 and its high-affinity receptor. Following this upregulation, the expression of NGF and TrkA are inhibited through the NT-3-TrkC pathway, inhibiting the downstream signaling and expression of the mediators induced by the NGF-TrkA pathway. However, the underlying mechanism needs further investigation. Together, this study demonstrates that the analgesic effect of oxycodone may be achieved through the activation of MOR downstream signaling, attenuating glial activation, and fortifying antinociceptive mediators in the spinal cord. Nevertheless, there are some limitations to our research. We did not further explore the possible mechanism underlying the effect of oxycodone on NTs and receptors, which should be investigated in future studies.

\section{Conclusion}

The results of this study revealed that the antinociceptive properties of oxycodone in a rat model of acute post-surgery pain can be attributed to the activation of MOR downstream signaling rather than KOR or DOR. The results of this study also suggested that the analgesic effect of oxycodone may be attributed to the regulation of the expression of NTs and their receptors in the spinal cord. The suppression of pro-nociceptive molecules by oxycodone pretreatment provides another argument in favor of an early application of analgesic drugs to prevent post-surgery pain. This study, to a certain degree, will hopefully improve our understanding of the underlying mechanism of oxycodone in acute post-surgery pain and may provide new candidates for further investigations into the mechanism underlying the effects of oxycodone.

\section{Acknowledgment}

The authors thank Beijing Mundipharma Pharmaceutical Co., Ltd., for kindly providing oxycodone for this study.

\section{Author contributions}

All the authors contributed toward data analysis, drafting and revising the paper, and agree to be accountable for all aspects of the work.

\section{Disclosure}

The authors report no conflicts of interest in this work.

\section{References}

1. Argoff CE. Recent management advances in acute postoperative pain. Pain Pract. 2014;14(5):477-487.

2. DeLeo JA, Colburn RW, Nichols M, Malhotra A. Interleukin-6-mediated hyperalgesia/allodynia and increased spinal IL-6 expression in a rat mononeuropathy model. J Interferon Cytokine Res. 1996;16(9):695-700.

3. Watkins LR, Martin D, Ulrich P, Tracey KJ, Maier SF. Evidence for the involvement of spinal cord glia in subcutaneous formalin induced hyperalgesia in the rat. Pain. 1997;71(3):225-235.

4. Watkins LR, Milligan ED, Maier SF. Spinal cord glia: new players in pain. Pain. 2001;93(3):201-205.

5. Ashton JC. Neuropathic pain: an evolutionary hypothesis. Med Hypotheses. 2012;78(5):641-643.

6. Mika J, Zychowska M, Popiolek-Barczyk K, Rojewska E, Przewlocka B. Importance of glial activation in neuropathic pain. Eur J Pharmacol. 2013;716(1-3):106-119.

7. Chapman CR, Vierck CJ. The Transition of Acute Postoperative Pain to Chronic Pain: An Integrative Overview of Research on Mechanisms. J. Pain. 2017;4(18):359 e1-e38.

8. Ha SO, Kim JK, Hong HS, Kim DS, Cho HJ. Expression of brain-derived neurotrophic factor in rat dorsal root ganglia, spinal cord and gracile nuclei in experimental models of neuropathic pain. Neuroscience. 2001;107(2):301-309.

9. Li CQ, Xu JM, Liu D, Zhang JY, Dai RP. Brain derived neurotrophic factor (BDNF) contributes to the pain hypersensitivity following surgical incision in the rats. Mol Pain. 2008;4:27.

10. Ceni C, Unsain N, Zeinieh MP, Barker PA. Neurotrophins in the regulation of cellular survival and death. Handb Exp Pharmacol. 2014;220:193-221.

11. Skaper SD. The neurotrophin family of neurotrophic factors: an overview. Methods Mol Biol. 2012;846:1-12.

12. Patapoutian A, Reichardt LF. Trk receptors: mediators of neurotrophin action. Curr Opin Neurobiol. 2001;11(3):272-280.

13. Spofford CM, Brennan TJ. Gene expression in skin, muscle, and dorsal root ganglion after plantar incision in the rat. Anesthesiology. 2012;117(1):161-172.

14. Wu C, Boustany L, Liang H, Brennan TJ. Nerve growth factor expression after plantar incision in the rat. Anesthesiology. 2007;107(1):128-135.

15. Banik RK, Subieta AR, Wu C, Brennan TJ. Increased nerve growth factor after rat plantar incision contributes to guarding behavior and heat hyperalgesia. Pain. 2005;117(1-2):68-76.

16. Ordóñez Gallego A, González Barón M, Espinosa Arranz E. Oxycodone: a pharmacological and clinical review. Clin Transl Oncol. 2007;9(5):298-307.

17. Anastassopoulos KP, Chow W, Tapia CI, et al. Economic study on the impact of side effects in patients taking oxycodone controlled-release for noncancer pain. J Manag Care Pharm. 2012;18(8):615-626.

18. Yoburn BC, Shah S, Chan K, Duttaroy A, Davis T. Supersensitivity to opioid analgesics following chronic opioid antagonist treatment: relationship to receptor selectivity. Pharmacol Biochem Behav. 1995;51(2-3):535-539.

19. Virk MS, Williams JT. Agonist-specific regulation of mu-opioid receptor desensitization and recovery from desensitization. Mol Pharmacol. 2008;73(4):1301-1308.

20. Nielsen CK, Ross FB, Lotfipour S, Saini KS, Edwards SR, Smith MT. Oxycodone and morphine have distinctly different pharmacological profiles: radioligand binding and behavioural studies in two rat models of neuropathic pain. Pain. 2007;132(3):289-300.

21. Lalovic B, Kharasch E, Hoffer C, Risler L, Liu-Chen LY, Shen DD. Pharmacokinetics and pharmacodynamics of oral oxycodone in healthy human subjects: role of circulating active metabolites. Clin Pharmacol Ther. 2006;79(5):461-479.

22. Hervera A, Negrete R, Leánez S, Martín-Campos JM, Pol O. Peripheral effects of morphine and expression of $\mu$-opioid receptors in the dorsal root ganglia during neuropathic pain: nitric oxide signaling. Mol Pain. 2011;7:25. 
23. Saloman JL, Niu KY, Ro JY. Activation of peripheral delta-opioid receptors leads to anti-hyperalgesic responses in the masseter muscle of male and female rats. Neuroscience. 2011;190:379-385.

24. Miranda JA, Stanley P, Gore K, Turner J, Dias R, Rees H. A preclinical physiological assay to test modulation of knee joint pain in the spinal cord: effects of oxycodone and naproxen. PLoS One. 2014;9(8):e106108.

25. Hassan HE, Myers AL, Lee IJ, Chen H, Coop A, Eddington ND. Regulation of gene expression in brain tissues of rats repeatedly treated by the highly abused opioid agonist, oxycodone: microarray profiling and gene mapping analysis. Drug Metab Dispos. 2010;38(1):157-167.

26. Zhang Y, Liang Y, Levran O, et al. Alterations of expression of inflammation/immune-related genes in the dorsal and ventral striatum of adult C57BL/6J mice following chronic oxycodone self-administration: a RNA sequencing study. Psychopharmacology (Berl). 2017;234(15):2259-2275.

27. Donnerer J, Liebmann I. Upregulation of BDNF and Interleukin-1ß in rat spinal cord following noxious hind paw stimulation. Neurosci Lett 2018;665:152-155.

28. Mousa SA, Cheppudira BP, Shaqura M, et al. Nerve growth factor governs the enhanced ability of opioids to suppress inflammatory pain. Brain. 2007;130(Pt 2):502-513.

29. Brennan TJ, Vandermeulen EP, Gebhart GF. Characterization of a rat model of incisional pain. Pain. 1996;64(3):493-501.

30. Stubhaug A, Breivik H, Eide PK, Kreunen M, Foss A. Mapping of punctuate hyperalgesia around a surgical incision demonstrates that ketamine is a powerful suppressor of central sensitization to pain following surgery. Acta Anaesthesiol Scand. 1997;41(9):1124-1132.

31. Martinez V, Fletcher D, Bouhassira D, Sessler DI, Chauvin M. The evolution of primary hyperalgesia in orthopedic surgery: quantitative sensory testing and clinical evaluation before and after total knee arthroplasty. Anesth Analg. 2007;105(3):815-821.

32. Xu J, Richebe P, Brennan TJ. Separate groups of dorsal horn neurons transmit spontaneous activity and mechanosensitivity one day after plantar incision. Eur J Pain. 2009;13(8):820-828.

33. Pogatzki EM, Gebhart GF, Brennan TJ. Characterization of Adelta- and C-fibers innervating the plantar rat hindpaw one day after an incision. J Neurophysiol. 2002;87(2):721-731.

34. Angst MS, Clark JD. Opioid-induced hyperalgesia: a qualitative systematic review. Anesthesiology. 2006;104(3):570-587.

35. Pöyhiä R, Kalso EA. Antinociceptive effects and central nervous system depression caused by oxycodone and morphine in rats. Pharmacol Toxicol. 1992;70(2):125-130.

36. Mills CD, Nguyen T, Tanga FY, et al. Characterization of nerve growth factor-induced mechanical and thermal hypersensitivity in rats. Eur $J$ Pain. 2013;17(4):469-479.

37. Osikowicz M, Longo G, Allard S, Cuello AC, Ribeiro-da-Silva A. Inhibition of endogenous NGF degradation induces mechanical allodynia and thermal hyperalgesia in rats. Mol Pain. 2013;9:37.

38. Mousa SA, Zhang Q, Sitte N, Ji R, Stein C. beta-Endorphin-containing memory-cells and mu-opioid receptors undergo transport to peripheral inflamed tissue. J Neuroimmunol. 2001;115(1-2):71-78.

39. Zollner C, Shaqura MA, Bopaiah CP, Mousa S, Stein C, Schafer M. Painful inflammation-induced increase in mu-opioid receptor binding and G-protein coupling in primary afferent neurons. Mol Pharmacol. 2003;64(2):202-210.

40. Shaqura MA, Zöllner C, Mousa SA, Stein C, Schäfer M. Characterization of $\mathrm{mu}$ opioid receptor binding and $\mathrm{G}$ protein coupling in rat hypothalamus, spinal cord, and primary afferent neurons during inflammatory pain. J Pharmacol Exp Ther 2004;308(2):712-718.

41. Sun Y, Sahbaie P, Liang D, et al. DNA Methylation Modulates Nociceptive Sensitization after Incision. PLoS One. 2015;10(11):e0142046.

42. Nakamura A, Hasegawa M, Minami K, et al. Differential activation of the mu-opioid receptor by oxycodone and morphine in painrelated brain regions in a bone cancer pain model. Br. J. Pharmacol. 2013;168(2):375-388.
43. Lemberg KK, Kontinen VK, Siiskonen AO, et al. Antinociception by spinal and systemic oxycodone: why does the route make a difference? In vitro and in vivo studies in rats. Anesthesiology. 2006;105(4):801-812.

44. Wang TH, Meng QS, Qi JG, Zhang WM, Chen J, Wu LF. NT-3 expression in spared DRG and the associated spinal laminae as well as its anterograde transport in sensory neurons following removal of adjacent DRG in cats. Neurochem Res. 2008;33(1):1-7.

45. Wilson-Gerwing TD, Dmyterko MV, Zochodne DW, Johnston JM, Verge VM. Neurotrophin-3 suppresses thermal hyperalgesia associated with neuropathic pain and attenuates transient receptor potential vanilloid receptor-1 expression in adult sensory neurons. J Neurosci. 2005;25(3):758-767.

46. Mantyh PW, Koltzenburg M, Mendell LM, Tive L, Shelton DL. Antagonism of nerve growth factor-TrkA signaling and the relief of pain. Anesthesiology. 2011;115(1):189-204.

47. Andreev NYu, Dimitrieva N, Koltzenburg M, McMahon SB. Peripheral administration of nerve growth factor in the adult rat produces a thermal hyperalgesia that requires the presence of sympathetic post-ganglionic neurones. Pain. 1995;63(1):109-115.

48. Dyck PJ, Peroutka S, Rask C, et al. Intradermal recombinant human nerve growth factor induces pressure allodynia and lowered heat-pain threshold in humans. Neurology. 1997;48(2):501-505.

49. McKelvey L, Shorten GD, O’Keeffe GW. Nerve growth factormediated regulation of pain signalling and proposed new intervention strategies in clinical pain management. J Neurochem. 2013;124(3): 276-289.

50. Kerr BJ, Bradbury EJ, Bennett DL, et al. Brain-derived neurotrophic factor modulates nociceptive sensory inputs and NMDA-evoked responses in the rat spinal cord. J Neurosci. 1999;19(12):5138-5148.

51. Pezet S, McMahon SB. Neurotrophins: mediators and modulators of pain. Annu Rev Neurosci. 2006;29:507-538.

52. Yajima Y, Narita M, Narita M, Matsumoto N, Suzuki T. Involvement of a spinal brain-derived neurotrophic factor/full-length TrkB pathway in the development of nerve injury-induced thermal hyperalgesia in mice. Brain Res. 2002;958(2):338-346.

53. Heppenstall PA, Lewin GR. BDNF but not NT-4 is required for normal flexion reflex plasticity and function. Pro Nat Acad Sci. 2001;98(14):8107-8112.

54. Buck CR, Seburn KL, Cope TC. Neurotrophin expression by spinal motoneurons in adult and developing rats. J Comp Neurol. 2000;416(3):309-318.

55. Stephens HE, Belliveau AC, Gupta JS, Mirkovic S, Kablar B. The role of neurotrophins in the maintenance of the spinal cord motor neurons and the dorsal root ganglia proprioceptive sensory neurons. Int J Dev Neurosci. 2005;23(7):613-620.

56. Funakoshi H, Frisén J, Barbany G, et al. Differential expression of mRNAs for neurotrophins and their receptors after axotomy of the sciatic nerve. J Cell Biol. 1993;123(2):455-465.

57. Joseph EK, Levine JD. Mu and delta opioid receptors on nociceptors attenuate mechanical hyperalgesia in rat. Neuroscience. 2010;171(1):344-350.

58. Woolf CJ, Mannion RJ. Neuropathic pain: aetiology, symptoms, mechanisms, and management. Lancet. 1999;353(9168):1959-1964.

59. Streit WJ, Graeber MB, Kreutzberg GW. Functional plasticity of microglia: a review. Glia. 1988;1(5):301-307.

60. Kato H, Walz W. The initiation of the microglial response. Brain Pathol. 2000;10(1):137-143.

61. Zhang XM, Zhu J. Kainic Acid-induced neurotoxicity: targeting glial responses and glia-derived cytokines. Curr Neuropharmacol. 2011;9(2):388-398.

62. Yang PP, Yeh GC, Huang EY, Law PY, Loh HH, Tao PL. Effects of dextromethorphan and oxycodone on treatment of neuropathic pain in mice. J Biomed Sci. 2015;22(1):81.

63. Ye J, Yan H, Xia Z. Oxycodone ameliorates the inflammatory response induced by lipopolysaccharide in primary microglia. J Pain Res. 2018;11:1199-1207 
64. Siuciak JA, Altar CA, Wiegand SJ, Lindsay RM. Antinociceptive effect of brain-derived neurotrophic factor and neurotrophin-3. Brain Res. 1994;633(1-2):326-330.

65. Siuciak JA, Wong V, Pearsall D, Wiegand SJ, Lindsay RM. BDNF produces analgesia in the formalin test and modifies neuropeptide levels in rat brain and spinal cord areas associated with nociception. Eur $J$ Neurosci. 1995;7(4):663-670.
66. Khan N, Smith MT. Neurotrophins and Neuropathic Pain: Role in Pathobiology. Molecules. 2015;20(6):10657-10688.

67. Malcangio M, Garrett NE, Cruwys S, Tomlinson DR. Nerve growth factor- and neurotrophin-3-induced changes in nociceptive threshold and the release of substance $\mathrm{P}$ from the rat isolated spinal cord. J Neurosci. 1997;17(21):8459-8467.

\section{Publish your work in this journal}

The Journal of Pain Research is an international, peer reviewed, open access, online journal that welcomes laboratory and clinical findings in the fields of pain research and the prevention and management of pain. Original research, reviews, symposium reports, hypothesis formation and commentaries are all considered for publication.
Dovepress

The manuscript management system is completely online and includes a very quick and fair peer-review system, which is all easy to use. Visit http://www.dovepress.com/testimonials.php to read real quotes from published authors. 